\title{
The Effect of Visual Feedback on Body Sway in the Elderly with Diabetes Compared to Elderly without Diabetes: Randomized Clinical Trial
}

\author{
Faris S. Alshammari ${ }^{1,2 *}$, Jerrold S. Petrofsky ${ }^{3}$, Noha S. Daher ${ }^{4}$, Salem O. Dehom ${ }^{5}$ and Eman S. \\ Alzoghbieh ${ }^{6}$ \\ ${ }^{1}$ Doctor of Physical Therapy Program, University of St Augustine for Health Sciences, San Marcos, CA \\ ${ }^{2}$ Department of Physical and Occupational Therapy, The Hashemite University, Zarqa, Jordan \\ ${ }^{3}$ School of Physical Therapy, Touro University, Henderson, NV
}

4Department of Allied Health Studies, School of Allied Health Sciences, Loma Linda University, Loma Linda, CA

${ }^{5}$ School of Nursing, Loma Linda University, Loma Linda, CA

${ }^{6}$ School of Natural Sciences and Human Ecology, California State University, San Bernardino, CA

*Corresponding author: Faris Alshammari, Doctor Physical Therapy Program, University of St Augustine for Health Sciences, San

Marcos, CA

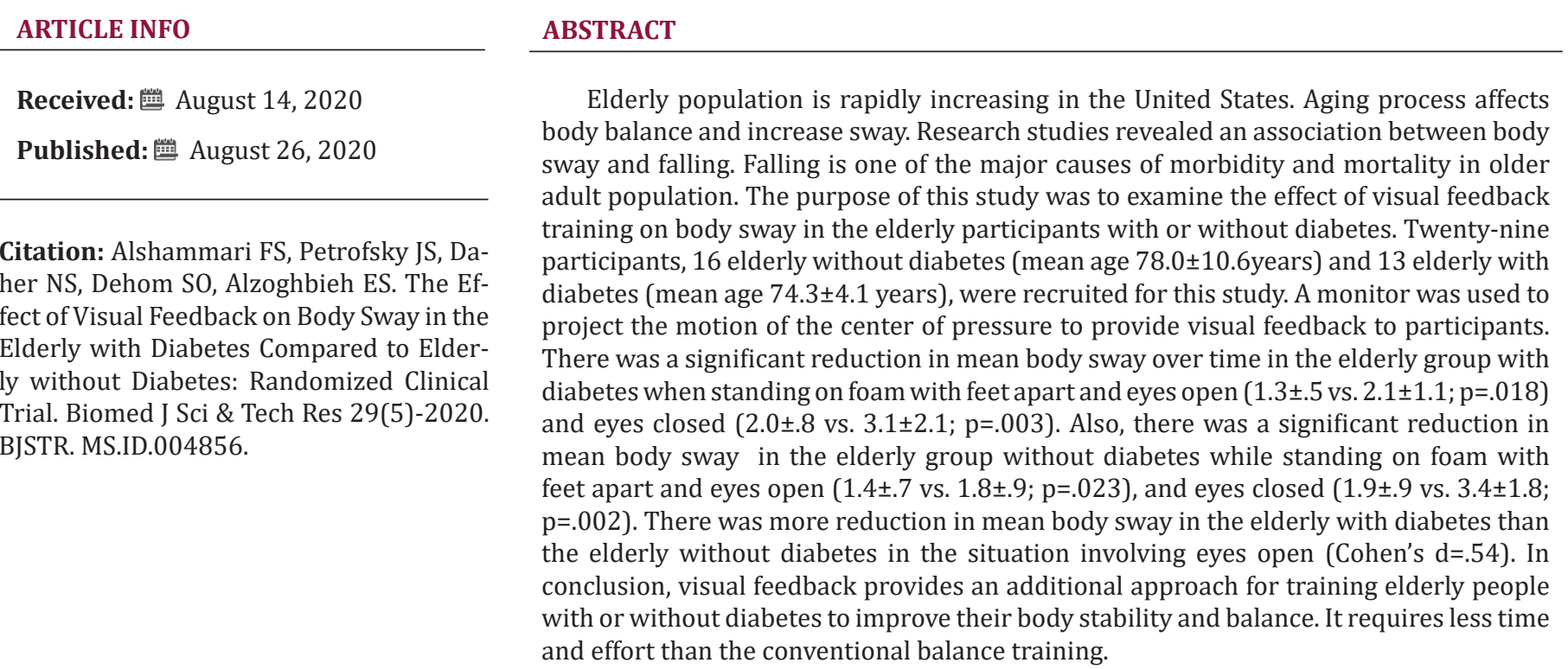

\section{Introduction}

Balance is a complicated mechanism aiming to maintain body posture during the presence of static and dynamic stressors [1]. The balance system has 3 main components. The first component is the input including vision, the vestibular system, and the somato sensory system. The second component is the central processing of the received data. Central processing involves many pathways and integration between different areas in the cerebellum, cerebrum, and spinal cord [1]. The third component is the output which is projected through the musculoskeletal system. Deficits in any of these components or poor coordination between these components can result in postural instability indicated by an increase in body sway and increased risk of falling [1,2]. The elderly population 
is rapidly increasing in the United States (US). According to the Department of Health and Human Services, in the year 2009, the number of people who were 65 years or older was 39.6 million. However, by the year 2030, the projected number of people who are 65 years or older will be around 72.1 million [3].

Balance disorders are presented more in the elderly compared to young people due to the aging process and high incidence of diseases such as diabetes [4-6]. Aging involves many physiological changes in the sensory, visual, vestibular, motor, and central nervous systems (CNS) resulting in poor postural control [4] A previous study has shown a significant indirect relationship between visual dysfunction and body stability [7]. This can be seen either as increased body sway, decreased postural control, or sustained falls among the elderly [2]. Studies have demonstrated a direct relationship between increasing body sway in the elderly and the risk of falling [2,8]. Falling is one of the major causes of morbidity and mortality in the elderly population. According to the Center for Disease Control and Prevention (CDC), 1 out of 3 adults who are 65 years or older fall every year [9]. In the year of 2010, 2.3 million falls related nonfatal injuries among elderly were reported and 662,000 of these fallers were hospitalized [9]. In the same year, the medical cost associated with these injuries was 30 billion dollars [9].

The prevalence of diabetes has increased dramatically over the last 20 years. According to the World Health Organization, there were 171 million people affected with diabetes in the year 2000 . However, the projected increase in the incidence of diabetes by the year 2030 will be 366 million people [10]. Also, according to the U.S Department of Health and Human Services, in 2010, the prevalence of diabetes in people 65 years or older was $26.9 \%$, affecting 10.9 million people [11]. Uncontrolled diabetes affects body balance significantly by affecting sensory inputs, brain function, and/or motor control [12]. The prevalence of peripheral neuropathy is between $60-70 \%$ among people with diabetes [12]. Loss of sensory input or muscle strength in peripheral neuropathy affects the postural stability and body balance significantly $[2,13,14]$. Kim and colleagues reported that $60 \%$ of patients with diabetic neuropathy develop vestibular disorders [15]. Another study showed that the vestibular disorders increased the risk of falls over 2 fold in people with diabetes [16]

Song and colleagues [17] examined the effect of an exercise program on balance and proprioception in the elderly participants with peripheral neuropathy. They used a balance training program for 60 minutes twice a week for 8 weeks. They found a significant reduction in postural sway following the training program [17]. Cawsey and colleagues [18] tested the effect of center of pressure (COP) magnification using visual feedback on standing sway. They found that the standing sway on incompliant and compliant surfaces decreased significantly with COP magnification [18]. In summary, body balance is affected by the aging process and the high incidence of disease in the elderly. The impact on body sway is more among the elderly with diabetes due to damage in the CNS, PNS, vestibular system, vision, and musculoskeletal system. Body sway is worse in balance disorders and studies showed a direct relationship between body sway and the incidence of falling in the elderly. Current regimen in balance training requires a long duration and increased repetition which could be challenging to the elderly due to limited fitness and presence of disease and health disorders. Therefore, the purpose of this study was to examine the effect of a single 6 minutes of visual feedback training on body sway in the elderly participants with or without diabetes.

\section{Methods \\ Participants}

Fifty-one participants were enrolled in this study. They were recruited using fliers and telephone calls from physical therapy centers, senior homes, sport centers, and Loma Linda University. The participants were included if they are 65-90 years of age, either having no diabetes or a type 2 diabetes, and having balance deficits presented by a complaint of instability. Subjects were divided into 2 groups; an elderly group without diabetes and an elderly group with diabetes. Then the participants were assigned randomly into 2 different interventions using a block design. One of these interventions was the visual feedback. Therefore, there were 13 participants in the elderly group with diabetes and 16 participants in the elderly group without diabetes receiving visual feedback. Participants were excluded if they had cognitive disorders, central nervous system disorders, alcohol/substance abuse, took medications that affected body balance, vestibular pathology, unable to stand for 10 minutes continuously, or enrolled in balance training that interfered with the provided intervention within the last month. All participants were screened with a $10 \mathrm{-g}$ monofilament test for neuropathy, and none of them failed the test. All protocols and procedures were approved by the Institutional Review Board of Loma Linda University and all participants signed a statement of informed consent. The rights of human subjects were protected. The baseline demographics of each group are shown in Table 1 . There was a significant difference in weight between the 2 study groups.

Table 1: Demographic data of the elderly subjects with and without diabetes (Mean \pm Standard Deviation).

\begin{tabular}{|c|c|c|c|}
\hline & $\begin{array}{c}\text { Elderly without } \\
\text { diabetes }(\mathbf{n = 1 6})\end{array}$ & $\begin{array}{c}\text { Elderly with } \\
\text { diabetes } \mathbf{( n = 1 3 )}\end{array}$ & P\# value \\
\hline Age (years) & $78.0 \pm 10.6$ & $74.3 \pm 4.1$ & .11 \\
\hline Height $(\mathrm{cm})$ & $163.3 \pm 9.5$ & $167.6 \pm 11.2$ & .29 \\
\hline Weight $(\mathrm{kg})$ & $71.5 \pm 15.2$ & $98.1 \pm 28.3$ & .007 \\
\hline BMI* $\left.^{*} \mathrm{~kg} / \mathrm{m}^{2}\right)$ & $26.7 \pm 4.8$ & $34.5 \pm 7.8$ & .005 \\
\hline
\end{tabular}

*BMI $=$ Body Mass Index $=$ Weight $(\mathrm{Kg}) /$ Height $\left(\mathrm{m}^{2}\right)$

\#Independent t-test 


\section{Study Design}

A single blinded randomized clinical trial design was used. The participants were assigned randomly using a block design into visual feedback or another intervention. For the purpose of this paper, we are focusing on the effect of visual feedback on body sway.

\section{Measurements of Body Sway}

Measurements of body sway were conducted by a licensed physical therapist that had 5 years of experience in using the BioPac system. The displacement of the participants' center of pressure (COP) was measured using a balance platform of $1 \mathrm{~m}^{2}$ in size and $10 \mathrm{~cm}$ in height. Four stainless steel bars with 16 strain gauges were mounted at the 4 corners under the platform's surface (TML Strain Gauge FLA-6, 350-17, Tokyo, Japan). The output of the 4 Wheatstone strain gauge bridges was amplified with a BioPac $100 \mathrm{C}$ low-level bio potential amplifier and recorded on a BioPac Mp-150 system through a 24-bit A/D converter. The sampling rate was 2000 samples per second [19]. Sway was presented as a line on a screen detecting the magnitude and angular displacement of the body. The magnitudes of $\mathrm{X}$ and $\mathrm{Y}$ coordinates of the participant's center of pressure were used to calculate the displacement of the center of mass of exerted body load on the platform. Mean (M) and standard deviation (SD) were obtained by finding the average of the sway magnitude of $Y$ and $X$ over an interval of 6 seconds. Data extraction and entry was conducted by a trained physical therapist who was not aware of the participants' allocation into groups.

\section{Design of Visual Feedback Intervention}

The center of pressure (COP) was presented at the screen as a red dot. At first, the person stood on the platform and centered the dot in a square in the middle of the screen. They were then instructed to hold the dot as closely as possible in the square in the center. The movement of red dot was in the same direction of body sway. If they leaned left or right or forward or backward, the dot would move appropriately out of the box in the center. This provided the participants with a visual cue to stop swaying and hold the COP steady. The visual feedback system is represented in Figure 1.

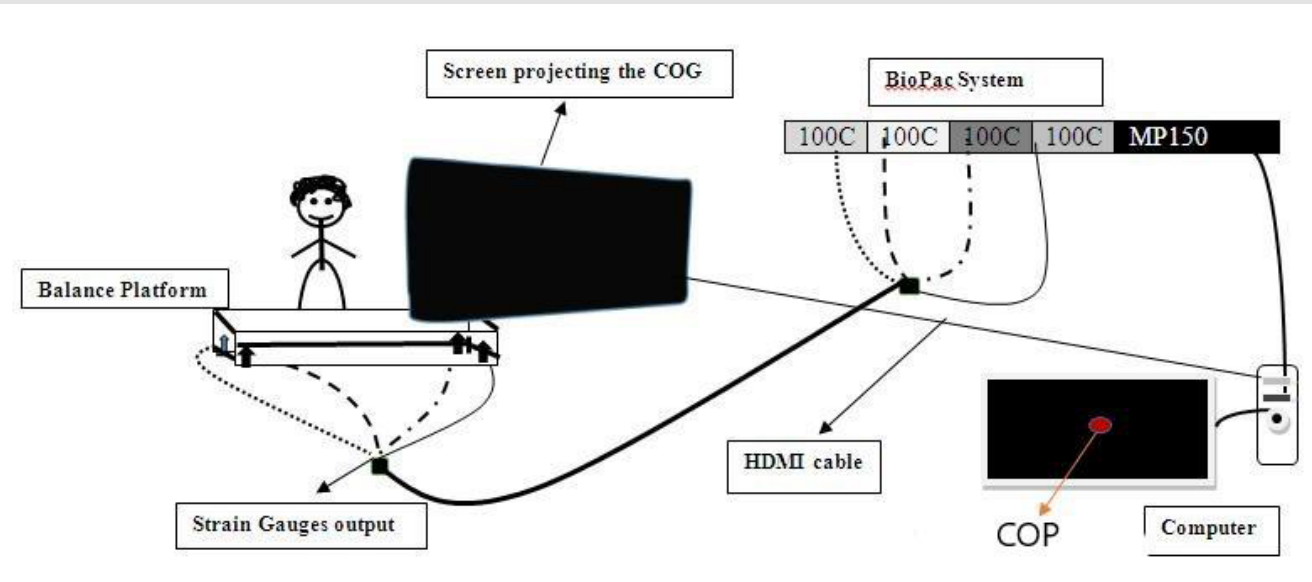

Figure 1: Visual feedback intervention.

\section{Balance Tasks}

Four balance tasks, with duration of 10 seconds, were included in this study. Two surface compliances (firm surface and foam) were used. Airex Balance Pad (SPRI Products Inc., Libertyville, IL, USA) was used in this study. The trial was conducted in a darkened room to increase the challenge of the tasks. The amount of the light in the room was measured using Light Meter model 615 (BK Precision Corporation, Yorba Linda, CA, USA). The light in the room was kept at 00.7 Lux while conducting the study. The four balance tasks were:

a) Standing on a firm surface (platform) with feet apart and eyes open;

b) Standing on a firm surface (platform) with feet apart and eyes closed; c) Standing on compliant surface (foam) with feet apart and eyes open; and

d) Standing on compliant surface (foam) with feet apart and eyes closed.

\section{Procedures}

The participants were asked to stand quietly on the platform with eyes open for 10 seconds. Then, the participants were asked to stand quietly on the platform with eyes closed for 10 seconds followed by 1 minute of rest. Following the 1 minute of rest, the same procedures were repeated again while the participants stood on foam. After finishing the baseline measurements, each participant received the visual feedback intervention. Visual feedback was given for 6 minutes divided into 3 sessions; 2 minutes each with 2 minutes of rest between them. If the participant swayed during the training session, he/she would see the motion of a red 
dot which representing the COP to give the participant cue to stand steadier. During the first training session, the participant was asked to hold the red dot as steady as possible by holding the body still while standing on platform (non-compliant surface). In the second training session, the sensitivity of the red dot to COP movement was increased by $50 \%$ and same procedures in first training session were repeated. In the third session, the participant was asked to stand on foam (compliant surface) and keep the red dot steady as much as possible by holding still. The same sensitivity of the COP in first training session was used in the third training session. Post intervention measurements were conducted following 2 minutes of rest after the third training session. Then, the participant was given 5 minutes of rest. Following that, another post intervention measurement was conducted to find the short term carryover of the intervention. Post intervention measures were the same as the measures prior to the intervention.

\section{Data Analysis}

The general characteristics of 16 elderly participants without diabetes and 13 elderly participants with diabetes were summarized using means and standard deviations for quantitative variables, and frequencies and relative frequencies for categorical variables. There was no attrition in the study. A 2x3 mixed factorial analysis of variance (ANOVA) with adjustment to the BMI were used to examine the difference in mean body sway between and within participants for the variables which were normally distributed. The differences between post and pre intervention measurements were compared between the two groups using independent t-tests. Effect sizes (Cohen's d) were calculated from the mixed models. Nonparametric analyses using Friedman and Wilcoxon Signed Rank tests were used to examine the difference in mean body sway within groups for the variables which were not normally distributed. The level of significance was set at $\mathrm{p} \leq .05$.

\section{Results}

There were no significant differences in mean body sway between the elderly group without diabetes and the elderly group with diabetes on any of the tasks pre intervention, post intervention, or 7 minutes post intervention. Body sway decreased significantly in all tasks that involved using foam following 6 minutes of visual feedback training. The average body sway decreased significantly in the elderly group with diabetes when standing on foam with feet apart and eyes open post intervention compared to pre intervention (1.3 \pm .5 vs. $2.1 \pm 1.1 ; p=.02$, Figure 2$)$. The average body sway decreased significantly in the elderly group with diabetes when standing on foam with feet apart and eyes closed post intervention compared to pre intervention ( $2.0 \pm .8$ vs. $3.1 \pm 2.1$; $p=.00$, Figure 3 ). The average body sway decreased significantly in the elderly group without diabetes while standing on foam with feet apart and eyes open post intervention compared to pre intervention (1.4 \pm .7 vs. $1.8 \pm .9 ; \mathrm{p}=.02$, Figure 4). Also, the average body sway decreased significantly in the elderly group without diabetes when standing on foam with feet apart and eyes closed post intervention when compared to pre intervention ( $1.9 \pm .9$ vs. $3.4 \pm 1.8$; $p=.00$, Figure 5). However, there was no significant difference in mean body sway between immediate post intervention measures and 7 minutes post intervention measures. There was a borderline significant difference in mean body sway difference while standing on foam

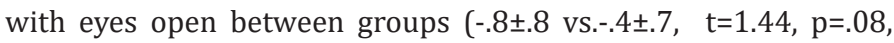
Cohen's $d=0.54)$. However this difference was not significant with eyes closed ( $\mathrm{t}=-.67, \mathrm{p}=.25$, Cohen's $\mathrm{d}=0.24$ ).

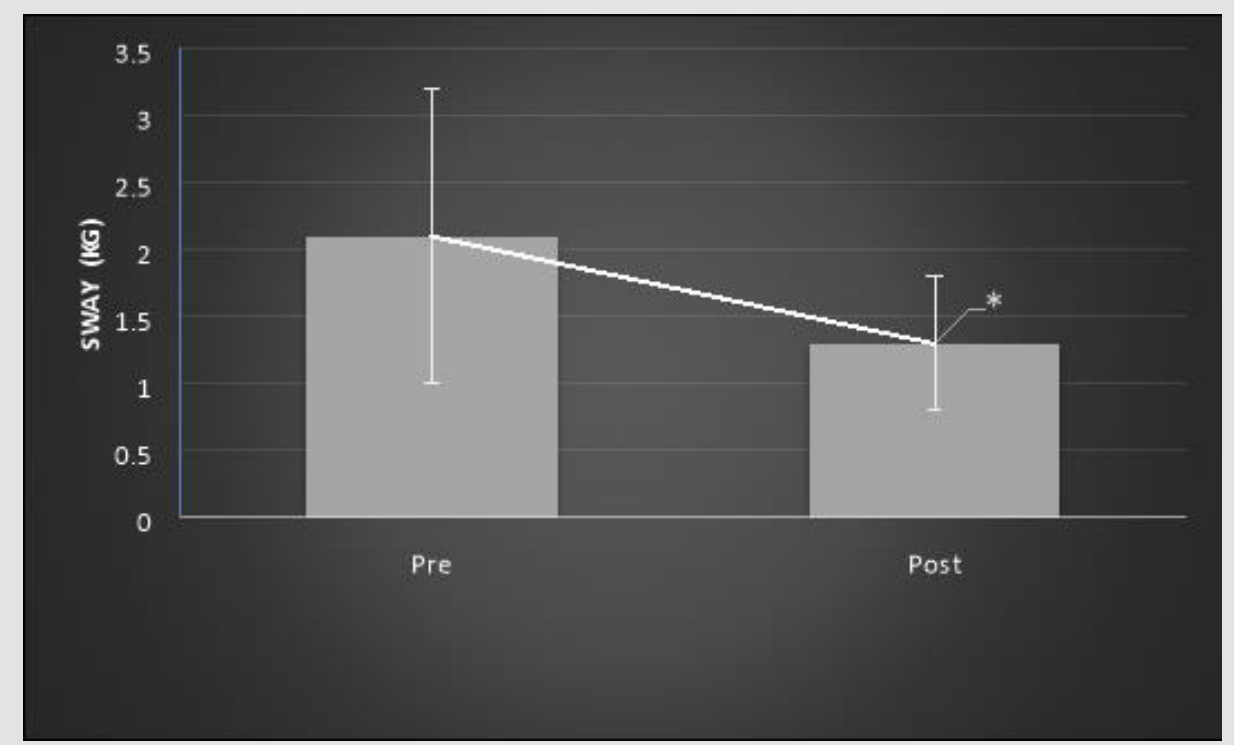

Figure 2: Mean body sway (in $\mathrm{kg}$ ) $\pm \mathrm{SD}$ while standing on foam with eyes open in elderly group with diabetes pre- and postintervention. ${ }^{*} \mathrm{p}=0.02$. 


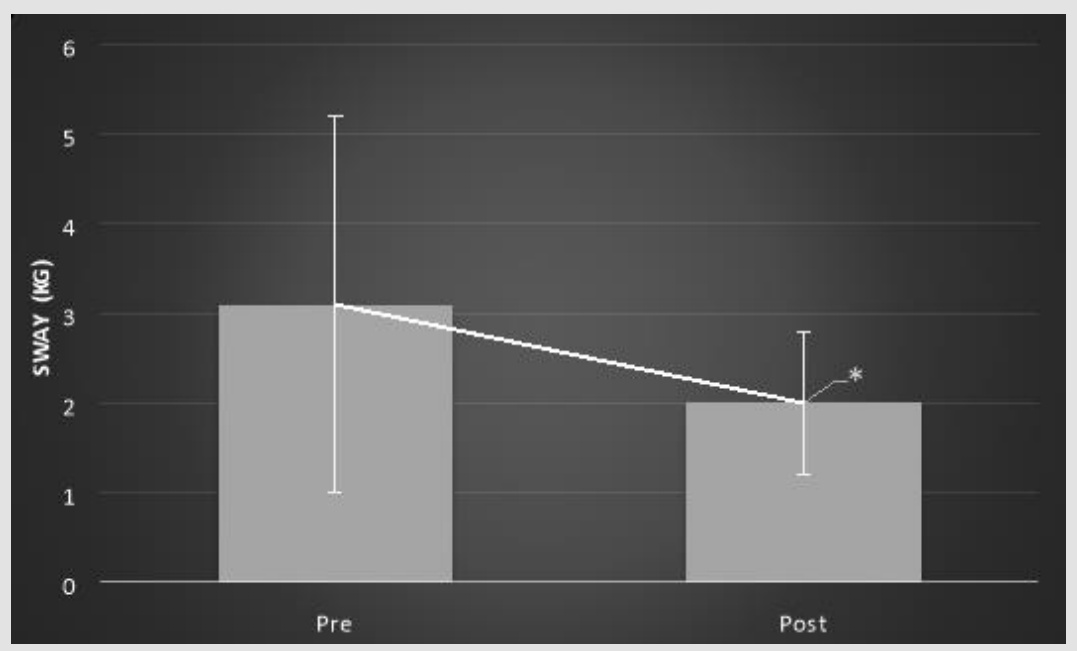

Figure 3: Mean body sway (in $\mathrm{kg}$ ) \pm SD while standing on foam with eyes closed in elderly group with diabetes pre- and postintervention. ${ }^{*} \mathrm{p}<.001$.

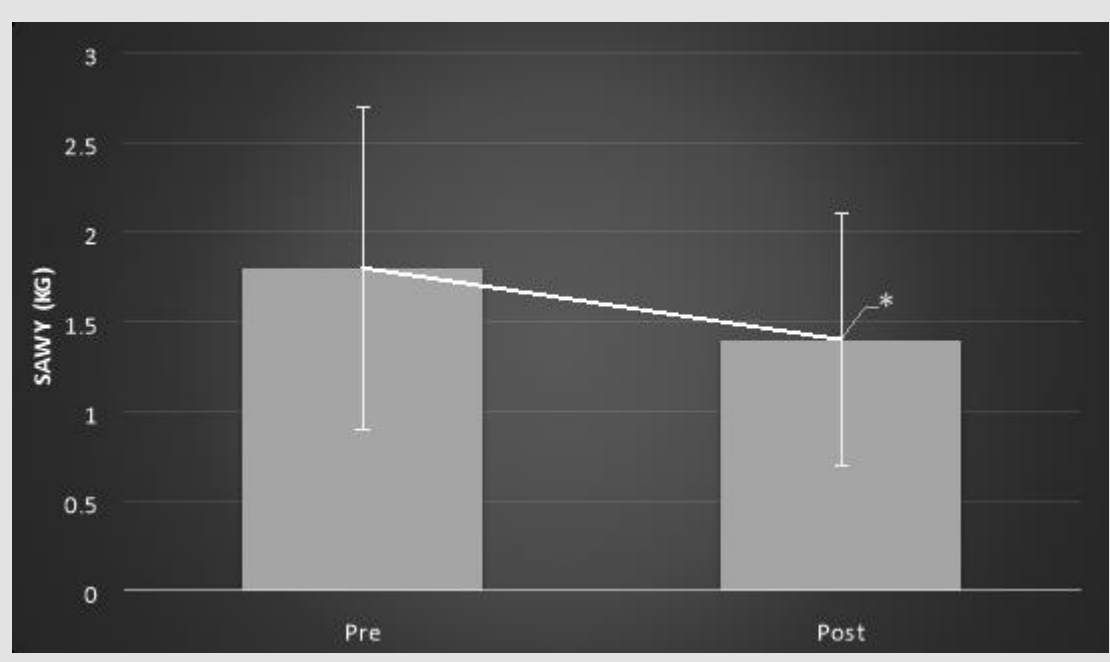

Figure 4: Mean body sway (in $\mathrm{kg}) \pm \mathrm{SD}$ while standing on foam with eyes open in elderly group without diabetes pre- and post- intervention. ${ }^{*} \mathrm{p}=0.02$.

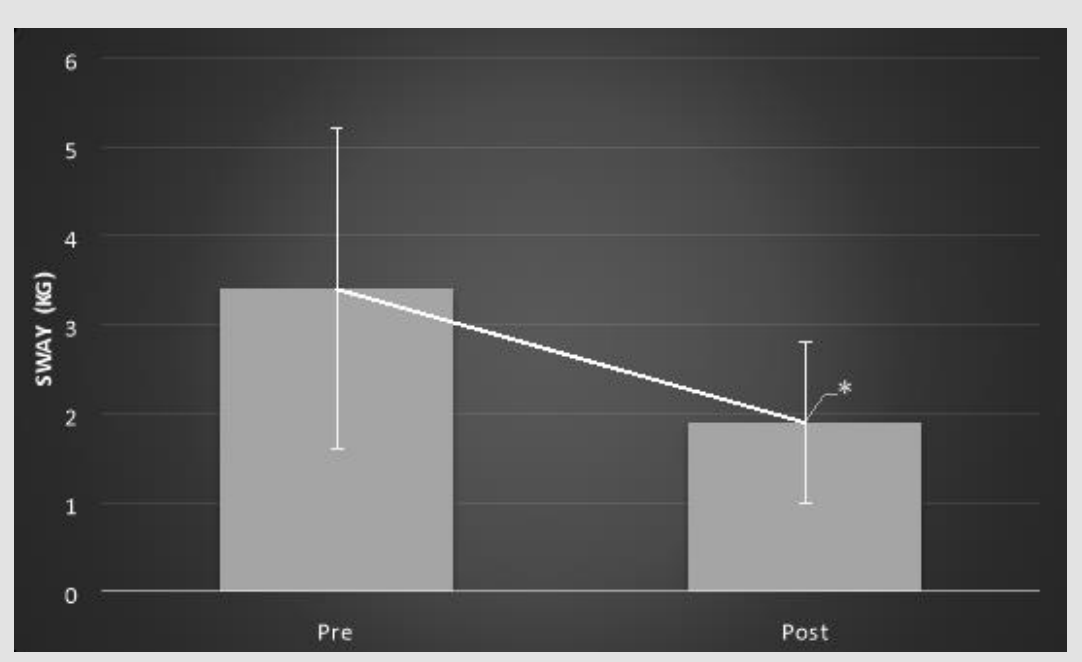

Figure 5: Mean body sway (in $\mathrm{kg}$ ) \pm SD while standing on foam with eyes closed in elderly group without diabetes pre- and post- intervention. ${ }^{*} \mathrm{p}<.001$. 


\section{Discussion}

Body balance diminishes with aging. This is due to the normal aging process and the high incidence of diseases that interfere with body balance $[4-6,20]$. Balance disorders result in increased body sway, decreased postural control, and increased incidence of falls $[2,21]$. Falling is one of the major predisposing factors of morbidity and mortality in the older adult population. According to the Center of Disease Control and Prevention, 1 out of 3 people who are 65 years or older fall every year [9]. This puts a financial burden on countries to deal with fall related injuries due to the high costs $[9,22]$. Body balance mechanism consists of 3 main components; sensory input, central processing, and output. Sensory inputs are conveyed via vision, the somatosensory system, and the vestibular system. Central processing takes place in the central nervous system including the brain and spinal cord. The output components of body balance are projected throughout the musculoskeletal system to maintain postural control and to prevent falling.

Diabetes can significantly affect peripheral sensory input and can result in peripheral neuropathy. A study has shown association between peripheral neuropathy and vestibular disorders [16]. The results of this study indicated better improvement in body sway in the elderly group with diabetes when compared to the elderly without diabetes while standing on foam with eyes open but not with eyes closed. This finding could be attributed to many reasons. As cited previously, vision predominates the somato sensory and vestibular systems in body balance in healthy people [23]. We would expect that vision will be involved more in controlling body balance in people with diabetes, compared to healthy people, due to limited peripheral somato sensory and vestibular inputs $[16,23]$. This might explain the reason that the elderly with diabetes, in this study, achieved better improvement than the elderly without diabetes in the situation involving standing on foam with eyes open. They could have learned a strategy to control their body sway using their visual input since they have to rely on the vision more than somato sensory and vestibular systems.

These findings are supported by a previous study that examined the effect of tactile feedback on body sway [24]. The elderly group without diabetes had more improvement than the elderly group with diabetes. This suggests that the participants in the elderly group without diabetes responded better to tactile feedback because they had better peripheral somato sensory input than the participants in the elderly group with diabetes [24]. Petrofsky and colleagues conducted a study to assess the motor control during balance tasks in participants with diabetes [25]. They found more activation in parietal cortex of the cerebrum in participants with diabetes compared to elderly without diabetes or young participants doing the same task. Also, the activation of parietal area increased with increasing the complexity of task such as eliminating vision or decreasing the somato sensory input in people with diabetes.
This finding suggests possible deficits in the sensor motor process and the integration of sensory inputs in people with diabetes. Their findings support the findings of this study that the participants in the elderly group with diabetes were able to learn new strategy to improve body sway in the situations involved eyes open because it makes the sensor motor process and sensory integration in parietal area less complex.

Other studies support the findings of this study $[26,27]$ Positive effect of visual feedback on body balance in frail elderly women was reported by Sihvonen et al. [27]. In the same study, the compliance with visual feedback among participants was high $97.5 \%$ and participants were motivated to participate in the training. In another study, visual feedback training helped in decreasing the incidence of fall among frail older women [26]. This suggests a promising future for visual feedback to be a useful training tool in physical therapy or rehabilitation centers to improve body balance in the elderly people. The limitations of this study were the small sample size, the intensity of treatment, and the lack of measurements of carryover effects. Future studies are recommended to study multiple visual feedback training sessions on a larger sample size and to have follow up measures to examine the carry over effect of the intervention.

\section{Conclusion}

Visual feedback provides an additional approach for training elderly people with or without diabetes to improve their body stability and balance. It requires less time and effort than the conventional balance training.

\section{References}

1. Winter DA (1995) Human Balance and Posture control during standing and walking. Gait \& Posture 3(4): 139-214.

2. Overstall PW, Exton-Smith AN, Imms FJ, Johnson AL (1977) Falls in the elderly related to postural imbalance. Br Med J 1(6056): 261-264.

3. (2014) Department of Health \& Human Services Aoa. Aging Statistics.

4. Dhar HL (1998) Physiology of aging. Indian J Med Sci 52(11): 485-497.

5. Du Pasquier RA, Blanc Y, Sinnreich M, Landis T, Burkhard P, et al. (2003) The effect of aging on postural stability: a cross sectional and longitudinal study. Neurophysiol Clin 33(5): 213-218.

6. Pyykko I, Jantti P, Aalto H (1990) Postural control in elderly subjects. Age Ageing 19(3): 215-221.

7. Anand V, Buckley JG, Scally A, Elliott DB (2003) Postural stability changes in the elderly with cataract simulation and refractive blur. Invest Ophthalmol Vis Sci 44(11): 4670-4675.

8. Fernie GR, Gryfe CI, Holliday PJ, Llewellyn A (1982) The relationship of postural sway in standing to the incidence of falls in geriatric subjects. Age Ageing 11(1): 11-16.

9. (2014) Prevention CfDCa. Falls Among Older Adults: An Overview.

10. (2014) Organization WH. Country and regional data on diabetes.

11. (2014) SERVICES USDOHAH. Fast Facts on Diabetes.

12. Ronald Aubert ea (1995) Diabetes in America. $2^{\text {nd }}($ Edn): NIH. 
13. Oppenheim U, Kohen-Raz R, Alex D, Kohen-Raz A, Azarya M (1999) Postural characteristics of diabetic neuropathy. Diabetes Care 22(2): 328-332.

14. De Mettelinge TR, Calders P, Palmans T, Vanden Bossche L, Van Den Noortgate N, et al. (2013) Vibration perception threshold in relation to postural control and fall risk assessment in elderly. Disabil Rehabil 35(20): 1712-1717.

15. Kim SK, Lee KJ, Hahm JR, et al. (2012) Clinical significance of the presence of autonomic and vestibular dysfunction in diabetic patients with peripheral neuropathy. Diabetes Metab J 36(1): 64-69.

16. Agrawal Y, Carey JP, Della Santina CC, Schubert MC, Minor LB (2010) Diabetes, vestibular dysfunction, and falls: analyses from the National Health and Nutrition Examination Survey. Otol Neurotol 31(9): 14451450 .

17. Chang Ho Song JSP, Seung Won Lee, Kyoung Jin Lee, Jong Eun Yim (2011) Effects of an exercise program on balance and trunk proprioception in older adults with diabetic neuropathies.Diabetes Technol Ther 13(8): 803-811.

18. Cawsey RP, Chua R, Carpenter MG, Sanderson DJ (2009) To what extent can increasing the magnification of visual feedback of the centre of pressure position change the control of quiet standing balance? Gait Posture 29(2): 280-284.

19. Petrofsky JS (2006) A device for the evaluation of sitting and reach balance in people in wheelchairs and standing. J Med Eng Technol 30(6): 358-367.

ISSN: 2574-1241

DOI: 10.26717/BJSTR.2020.29.004856

Faris Alshammari. Biomed J Sci \& Tech Res

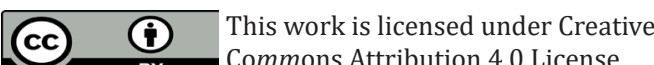

Submission Link: https://biomedres.us/submit-manuscript.php
20. Sturnieks DL, St George R, Lord SR (2008) Balance disorders in the elderly. Neurophysiol clin 38(6): 467-478.

21. Wolfson L, Whipple R, Derby CA, et al. (1992) A dynamic posturography study of balance in healthy elderly. Neurology 42(11): 2069-2075.

22. Stevens JA, Corso PS, Finkelstein EA, Miller TR (2006) The costs of fatal and non-fatal falls among older adults. Inj Prev 12(5): 290-295.

23. Grace Gaerlan M, Alpert PT, Cross C, Louis M, Kowalski S (2012) Postural balance in young adults: the role of visual, vestibular and somatosensory systems. J Am Acad Nurse Pract 24(6): 375-381.

24. Alshammari FS, Petrofsky JS, Daher N, Alzoghbieh ES, Dehom SO, et al. (2014) tactile intervention as a novel technique in improving body stability in healthy elderly and elderly with diabetes. Diabetes Technol Ther 16(12): 822-827.

25. Petrofsky JS, Alshammari F, Lee H, Jong YE, G Bains, et al. (2012) Electroencephalography to assess motor control during balance tasks in people with diabetes. Diabetes Technol Ther 14(11): 1068-1076.

26. Sihvonen S, Sipila S, Taskinen S, Era P (2004) Fall incidence in frail older women after individualized visual feedback-based balance training. Gerontology 50(6): 411-416.

27. Sihvonen SE, Sipila S, Era PA (2004) Changes in postural balance in frail elderly women during a 4-week visual feedback training: a randomized controlled trial. Gerontology 50(2): 87-95.

$\begin{array}{ll}\text { BIOMEDICAL } & \text { Assets of Publishing with us } \\ \text { RESEARCHES } & \text { - Global archiving of articles } \\ \text { - Immediate, unrestricted online access } & \text { - Rigorous Peer Review Process } \\ & \text { - Authors Retain Copyrights } \\ & \end{array}$

